\title{
Editorial: Tribology and Atomic Force Microscopy - Towards Single Asperity Contact
}

\author{
Brunero Cappella ${ }^{1 *}$, Dirk Spaltmann ${ }^{1}$ and Mark Gee ${ }^{2}$ \\ ${ }^{1}$ Federal Institute for Materials Research and Testing (BAM), Berlin, Germany, ${ }^{2}$ National Physical Laboratory, Teddington, \\ United Kingdom
}

Keywords: nanotribology, friction, wear, single asperity, AFM

\section{Editorial on the Research Topic}

Tribology and Atomic Force Microscopy - Towards Single Asperity Contact

The concept behind this Research Topic (RT) was to collect works, in which Atomic Force Microscopy (AFM) techniques are employed to study tribological phenomena and to push the resolution of measurements towards single asperity contact. Thanks to the direct determination of sample height with sub-nanometer resolution and the possibility of measuring local friction, AFM can be employed after a tribotest to detect topography and friction changes at the nanometer scale. Recently, efforts are being expended to use AFM cantilevers as tribometers, i.e., as probes altering the volume of suitable samples, thereby measuring tip and/or sample wear and friction at the nano/ microscale. Thus, single asperity contact, friction, and wear can be investigated. Since friction and wear at the macroscale are the result of asperities interactions, such experiments are of great importance for better understanding of tribological processes.

In the following, we briefly present the five papers comprising the RT.

The group of articles is opened by a study of Friedrich and Cappella, "Friction and Mechanical Properties of AFM-Scan-Induced Ripples in Polymer Films." It deals with periodic ripple patterns generated by AFM scanning in contact mode on polymer films, a well-known but not fully understood phenomenon. In this paper, AFM measurements are used to determine quantitatively the elastic modulus and the friction of the ripple structures with nanometer resolution.

The second article, "Micro- and Nanowear of Self-Mated Steel Generated and Studied with an AFM at the Single Asperity Level” by Reichelt and Cappella, presents for the first time tribological nanotests performed with self-mated steel. The AFM is used not only for the analysis of the wear of both tribopartners, but also for their generation, taking advantage of microscopic particles glued to the cantilever and employed as colloidal particles. The descriptive analysis of scars, wear particles, and wear of the colloidal particles impressively shows the capability of AFM in characterizing tribological phenomena with high spatial resolution down to single asperity events, thus enabling to characterize phenomena, which are of fundamental importance also at the macroscale. A second article dealing with scattering of data and dependence of the wear volume on the product of total sliding distance and load has been meanwhile published (Reichelt M., Cappella B., Wear Volume of Self-Mated Steel at the Submicron-Scale: An Atomic Force Microscopy Study, Journal of Tribology, 144, 061702-1-12, 2022).

The third contribution, "Statistics of Sliding on Periodic and Atomically Flat Surfaces" by Srbulovic et al., is a theoretical study of the application of the Prandtl-Tomlinson model to the numerical description of the sliding of the AFM tip over an atomically flat surface and of the resulting friction. 
Friction is again the subject of the 4th article published in this RT, "High-Velocity Shear and Soft Friction at the Nanometer Scale" by Thorén et al.. The authors investigate high-speed friction on soft polymers by means of AFM measurements. The result, specific to soft materials, show that an increase in velocity leads to a transition from stick-slip to free-sliding.

The last publication of this collection is "Oil as an Enabler for Efficient Materials Removal in Three-Dimensional Scanning Probe Microscopy Applications" by Noël et al.. In this work, the authors attempt to solve a problem arising when AFM tips are employed to wear a sample, namely wear of the tip itself and/or accumulation of wear particles at the tip. Through the comparison of tests in air and in oil environment, the authors show that oil can be used to prevent the accumulation of debris around the tip.

The editors thank all authors of this Research Topic.

\section{AUTHOR CONTRIBUTIONS}

All authors listed have made a substantial, direct, and intellectual contribution to the work and approved it for publication.

Conflict of Interest: The authors declare that the research was conducted in the absence of any commercial or financial relationships that could be construed as a potential conflict of interest.

Publisher's Note: All claims expressed in this article are solely those of the authors and do not necessarily represent those of their affiliated organizations, or those of the publisher, the editors and the reviewers. Any product that may be evaluated in this article, or claim that may be made by its manufacturer, is not guaranteed or endorsed by the publisher.

Copyright (C) 2022 Cappella, Spaltmann and Gee. This is an open-access article distributed under the terms of the Creative Commons Attribution License (CC BY).

The use, distribution or reproduction in other forums is permitted, provided the original author(s) and the copyright owner(s) are credited and that the original publication in this journal is cited, in accordance with accepted academic practice. No use, distribution or reproduction is permitted which does not comply with these terms. 